\title{
Study of Renal Artery Patterns in a Sri Lankan Population
}

Dissanayake $\mathrm{PH}^{1}$, Jayasekera $\mathrm{RW}^{2}$

${ }^{I}$ Department of Anatomy, Faculty of Medical Sciences, University of Sri Jayewardenepura.

${ }^{2}$ Department of Anatomy, Faculty of Medicine, University of Colombo.

\begin{abstract}
Introduction

The increased frequency of surgical as well as diagnostic and interventional radiological procedures involving the kidneys and the renal vasculature has shown a definite demand for a

re-evaluation of the renal arterial anatomy and its variations.
\end{abstract}

\section{Objective}

The objective of this study was to assess the anatomy of the renal arterial pattern in a Sri Lankan population.

\section{Methodology}

382 human cadaver kidneys were analyzed for its arterial anatomy.

\section{Results}

Of the 382 kidneys analysed, single renal arteries were found in 260 specimens (68.06\%) and 122 (31.94\%) had multiple renal arteries. The incidence of multiple renal arteries in males were $30.68 \%$ and $34.75 \%$. in females. A higher number of multiple renal arteries were found on the left, ie: 70 out of $122(57.38 \%)$ compared to 52 out of $122(42.62 \%)$ on the right.

A superior polar artery was given off from the main renal arteries in 86 kidneys (22.51\%) and an inferior polar artery was noted arising from the main renal artery in 29 kidneys $(7.59 \%)$.
Pre-hilar segmental branching was seen in $111(29.06 \%)$ of the main renal arteries.

\section{Conclusion}

$31.9 \%$ of our study population had multiple renal arteries. There was no significant difference with regard to the incidence of multiple renal arteries, between the two sexes nor on the sides. Prior knowledge of possible variations that may exist is important for the diagnostic and interventional radiologists, transplant surgeons and urologists. No detailed study has been done in a Sri Lankan population and in this regard our findings may provide some insight to the practising clinician in Sri Lanka.

\section{Introduction}

The incidence of diagnostic, therapeutic and surgical procedures involving the kidneys has increased in the recent past and the renal arterial anatomy and its variations do play a significant role in the above procedures. Hence there is a definite need to re-evaluate the normal renal arterial anatomy and its variations. $(1,2)$ Normally expected anatomy is to have a single renal artery supplying the hilum, but since 1552 when Eustachius first recorded anomalous or aberrant arteries, multiple variations have been 
documented with a broad spectrum of differences in the recorded literature in different study populations. $(3,4,5)$ Comprehensive anatomic dissections and arteriographic studies have shown that the simple form of renal arterial pattern occurs in less than half of individuals. $(5,6,7)$ The location and anatomy of the renal arteries is of importance for vascular surgeons, general surgeons, traumatologists, urologists and radiologists during surgical procedures and for diagnostic purposes, in order to arrive at a correct diagnosis and to perform surgery free of complication. Anomalous blood vessels are interesting to know from a scientific point of view, as especially they so often lead to the understanding of obscure problems of phylogeny and ontogeny.

The development of kidneys begins in the pelvis and migrate to its final adult location in the lumbar region. In this process they either retain blood vessels from their original location or receive and incorporate new vessels along its pathway to the final location. The presence of a range of accessory renal arteries originating from the pelvic vessels to the abdominal aorta is to be expected due to the persistence of the remains of embryonic renal vessels normally lost, in the process of development. $(10,11)$

\section{Methodology}

Ethical clearance was obtained from the Ethics review committee of the Faculty of Medicine, Colombo. Human cadaver kidneys were collected from the postmortems conducted at the Judicial Medical office, Colombo. The sample size was 382 human cadaver kidneys which were collected within 24 hours of the time of death. Specimens with diseased renal vessels, significantly small kidneys suggestive of renal vascular disease and patients with evidence of previous surgery in the related region were excluded. The specimens were carefully dissected and the morphology of the Reno-vascular system was analyzed and documented. The special morphological characteristics were noted, with regard to the renal arteries. The number of branches given off from the renal artery, presence or absence of the superior polar branch, presence or absence of the inferior polar branch and the presence or absence of the prehilar segmental branching were documented. The data was statistically analyzed utilizing the SPSS for windows software package.

\section{Results}

382 cadaver kidneys were analyzed where the age ranged from 7 years to 94 yrs. $118(30.9 \%)$ were from females and $264(69.1 \%)$ were from males. Of the 382 kidneys, single renal arteries were found in 260 specimens $(68.06 \%)$ and $122(31.94 \%)$ had multiple renal arteries.

The incidence of multiple renal arteries in males were $30.68 \%$ and $34.75 \%$. in females. A higher number of multiple renal arteries were found in the kidneys from the left side, ie: 70 out of 122 (57.38\%), compared to 52 out of 122 (42.62\%) from the right. 
When there were multiple renal arteries, 63 out of 122 (51.64\%), of the additional renal arteries that entered the hilum, 38 $(31.15 \%)$ entered the upper pole and 21 (17.21\%) entered the lower pole.

A superior polar artery was given off from the main renal arteries in 86 kidneys $(22.51 \%)$ and an inferior polar artery was noted arising from the main renal artery in 29 kidneys $(7.59 \%)$.

Two specimens had two superior polar branches arising from the single main renal artery.

Four specimens had superior polar branches originating from the additional renal arteries.

Pre-hilar segmental branching was seen in $111(29.06 \%)$ of the main renal arteries. Only three

additional renal arteries (2.29\%) had prehilar segmental branching.

\section{Discussion}

A search of the literature on the renal arterial anatomy and its variations in populations reveal a fair number of articles. Variations of the renal arterial pattern is becoming more relevant and important, with the increasing demand of diagnostic imaging, image guided therapeutic interventional procedures and surgical procedures such as renal transplantation. In addition vascular reconstructions involving renal arteries are becoming more commonplace in clinical and surgical practice. An awareness of the incidence of these variants in the general population in Sri Lanka will be of immensely beneficial in the surgical management of the aforementioned specialties.
In our study population we encountered $68.06 \%$ of kidneys with a single artery. When sex distribution of the single renal artery was analyzed, $69.32 \%$ of males and $65.25 \%$ of females had single renal arteries $(\mathrm{P}=0.609)$. Similarly $63.35 \%$ of left kidneys had a single renal artery and $72.78 \%$ on the right. $(\mathrm{P}=0.247)$. There was no significant correlation with the sex nor with the side of the kidney. Even though a wide variation in the points of origin has been described in the literature, we encountered all additional renal arteries arising from the abdominal aorta and none from the pelvic arteries nor from the branches of the aorta.

There is no unanimity in the nomenclature of renal arteries. The terms "extra", "aberrant", "ectopic" and "accessory", are commonly used when there are multiple arteries. Whatever the term that is used, the renal artery is considered to be an end artery and a damage to any renal artery regardless of its caliber, point of origin or destination will lead to ischaemia of the related renal tissue supplied by that particular artery. $(12,13)$

In 1954 Graves (14) demonstrated that once the renal artery enters the hilum, distribution of the arteries within the kidney substance was constant, namely the apical, upper, middle, lower and posterior five segmental branches. But the main stem of the renal artery may divides at any point between the aorta and the hilum, ie; pre-hilar segmental branching. In our study pre-hilar segmental branching was seen in $29.06 \%$ of the main renal arteries. Prehilar segmental branching was lower in similar studies done by Munnusamy 2016 and Ozkun 2006, 13\% and 8\% 
respectively. $(15,16)$ In our study, from the renal arteries that showed pre-hilar segmental branching, $39.47 \%$ were on the right side and $60.53 \%$ were on the left side. There was a significant relationship between the side and the pre-hilar segmental branching in our study $(\mathrm{P}=0.001)$ ie: occurrence more on the left but not in the previous 2 studies. $(15,16)$

Variations of renal arteries have been reported by several authors. Its prevalence range from $20 \%$ to $50 \%$ in their series. In our study, we encountered $31.94 \%$ of the kidneys having multiple renal arteries. The incidence of multiple renal arteries in males was $30.68 \%$ and in females were $34.75 \%$. $(\mathrm{P}=0.509)$. A higher number of multiple renal arteries were found in left kidneys, (36.65\%) compared to $(27.22 \%)$ on the right side. $(\mathrm{P}=0.586)$. There is no statistically significant difference of the multiple renal arteries depending on the sex nor the side. When the data is reviewed on the multiplicity of renal arteries, it seems that ethnicity and geographical location of the population could be an important factor on which the multiplicity of the renal arteries depend. $(3,5,6,7,17,18)$

When superior or inferior polar branches are present they are more prone to damage during mobilization of the kidneys or during other procedures. The inferior polar artery can compress the ureter causing obstruction. $(19,20)$ In our study $22.51 \%$ of the specimens had a superior polar branch given off from the main renal artery and $7.59 \%$ had an inferior polar branch given off from the main renal artery. Comparison of the incidence of superior and inferior polar branches were done with a similar study by Rupert RR (19, 20).

Table 1 - Incidence of the superior Polar Branch

\begin{tabular}{|l|l|l|}
\hline Side & Rupert RR $(\mathrm{n}=118)$ & Present series $(\mathrm{n}=382)$ \\
\hline Right & $24.0 \%$ & $14.4 \%$ \\
\hline Left & $14.0 \%$ & $9.5 \%$ \\
\hline
\end{tabular}

Table 2 - Incidence of the inferior Polar Branch

\begin{tabular}{|l|l|l|}
\hline Side & Rupert RR $(\mathrm{n}=118)$ & Present series $(\mathrm{n}=382)$ \\
\hline Right & $14.0 \%$ & $6.5 \%$ \\
\hline Left & $4.0 \%$ & $3.7 \%$ \\
\hline
\end{tabular}

Further, in our study, two specimens had two superior polar branches arising from the main renal artery.

Radiologically guided interventional procedures dealing with the kidneys are an integral part of the interventional radiologists work. Knowing the deviations from the normal anatomy of renal arteries in a respective population will prevent inadvertent damage, unexpected bleeding and other complications. Also being aware of the vascular variations one can impose restrictions in the area of exposure at surgery. Presence or absence of variations of the renal arterial pattern 
will influence the management plan of the vascular and transplant surgeon or the angiologist. $(1,2)$

Atherosclerotic renal artery stenosis is known to be a cause of systemic arterial hypertension and renal insufficiency. Percutaneous renal angioplasty and stenting is an accepted treatment modality, which gives a favourable response in ostial stenosis and improves blood pressure control, and delays or stabilizes renal insufficiency. Awareness of the variations in the anatomy of the renal arterial system is important in the handling of technical problems of percutaneous treatment of renal artery stenosis. (21)

Selective arteriography is used to evaluate renal lesions. However, it is important to inject all arteries whenever multiple renal arteries are present. If one of the renal segments is not filled with contrast in a kidney with multiple arteries, radiographically a single anatomic variant may lead to a wrong diagnosis of an infarct, avascular tumour or intrarenal haemorrhage. Its recognition may help to avoid a gross error in diagnosis. Angiographic evaluation of the kidney is incomplete without visualization of the entire renal vasculature and with the full renal parenchyma. $(22,23)$ Renal angiography is helpful in selecting donors for renal transplant surgery, in the evaluation of the functions of the diseased kidney, differentiation between cortical tumours and cystic disease, renal anomalies, congenital hypoplasia, chronic pyelonephritis, essential haematuria, ureteral obstruction and hypertension of doubtful aetiology. Once renal angiography is performed to interpret its findings, it is essential for the interventional radiologist to have a sound knowledge of the renal vasculature and its variations. Arteriography may also be considered before radical nephrectomy involving previous ligation of the renal artery, because the presence of multiple renal arteries may lead to failure in the control of the arterial supply before tumorous kidney mobilization. (12)

\section{Conclusion}

The topographic anatomy of the renal vasculature is subject to considerable variation. $31.9 \%$ of our study population had multiple renal arteries. There was no significant difference of having multiple renal arteries, between the two sexes nor on the sides. When the data is reviewed on the multiplicity of renal arteries, it seems that ethnicity and geographical location of the population could be an important factor which the multiplicity of the renal arteries depend on. Deviations from the normal anatomical pattern of arteries, and prior knowledge of possible variations that may exist, is important for the diagnostic and interventional radiologists, transplant surgeons and urologists who deal with kidneys. No detailed study has been done in a Sri Lankan population, and in this regard our findings may provide some insight to the practicing clinician in Sri Lanka. Also the variable morphology of renal arterial anatomy is of great interest to the anatomist and the embryologist. 


\section{References}

1. Makiyama K, Tanabe $\mathrm{K}$, Ishida $\mathrm{H}$, et al. Successful renovascular reconstruction for renal allografts with multiple renal arteries. Transplantation 2003; 75(6): 828832.

2. Aydin C, Berber I, Altaca G, et al. The outcome of kidney transplants with multiple renal arteries. BMC Surgery 2004; $4: 4$.

3. Anson BJ, Daseler ER. Common variations in renal anatomy, affecting blood supply, form and topography. Surgery Gynecology and Obstetrics 1961; 11:439-449.

4. Jeffery RF. Unusual origins of renal arteries. Radiology 1972; 102: 309310.

5. Anson BJ, Kurth LE. Common variations in the renal blood supply. Surgery Gynecology and Obstetrics 1955; 100: 156-162.

6. Merklin RJ, Michels NA. The variant renal and suprarenal blood supply with data on the inferior phrenic, ureteral and gonadal arteries. Journal of the International College of Surgeons 1958; 29:41-76.

7. Harrison LH Jr., Flye MW, Seigler HF. Incidence of anatomical variants in renal vasculature in the presence of normal renal function. Annals of Surgery 1978; 188: 83-89.

8. Dawson AB, Reis JH. An anomalous arterial supply to suprarenal, kidney and ovary. Anatomical Records 1922; 23: 161-167.
9. Ockerblad NF. Aberrant renal arteries which in themselves produce pain. The Journal of Urology 1952; 67: 810-814.

10. Bulie K, Ivkic G, Pavie T. A case of duplicated right renal artery and triplicated left renal artery. Annals of Anatomy 1996; 178: 281-283.

11. Roberts JBM. Congenital anomalies of the urinary tract and their association with spina bifida. British Journal of Urology 1961; 33: 309315.

12. Sampaio FJB, Passos MARF. Renal arteries: anatomic study for surgical and radiological practice. Surgical Radiologic Anatomy 1992; 14: 113117.

13. Graves FT. The arterial anatomy of congenitally abnormal kidney. British Journal of Surgery 1969; 56: 533-541.

14. Graves FT. The anatomy of the intrarenal arteries and its application to segmental resection of the kidney. The British Journal of Surgery 1954; 42: 132-139.

15. Munnusamy K, Kasirajan SP, Gurusamy $\mathrm{K}$ et al. Variations in Branching Pattern of Renal Artery in Kidney Donors Using CT Angiography. Journal of Clinical and Diagnostic Research. 2016 Mar, Vol10(3): AC01-AC03

16. Uğur Özkan, Levent Oğuzkurt, Fahri Tercan et al. Renal artery origins and variations: angiographic evaluation of 855 consecutive patients. Diagnostic and Interventional Radiology 2006; 12:183-186. 
17. Pick JW, Anson BJ. The renal vascular pedicle. An anatomical study of 430 body halves. The Journal of Urology 1940; 44: 411434.

18. Ross JA, Samuel E, Miller DR. Variations in the renal vascular pedicle. (an anatomical and radiological study with particular reference to renal transplantation). British Journal of Urology 1961; 33: 478-485.

19. Rupert RR. Further study of irregular kidney vessels as found in one hundred eighteen cadavers. Surgery Gynecology and Obstetrics 1915;21: 471-480.
20. Rupert RR. Irregular kidney vessels found in 50 cadavers. Surgery Gynecology and Obstetrics 1913; 17 : 580-585.

21. Howell MH, Strickman NE. Tandem Stenting of Crossed Renal Arteries with Ostial Stenosis. Texas Heart Institute Journal 2000; 27(2): 166169.

22. Siegelman SS, Agahan P, Barns LR. Incomplete renal angiography: the naked calyx sign. The Journal of Urology 1970; 103: 27-30.

23. Smith PG. A resume of the experiences in the making of 1500 renal angiograms. The Journal of Urology 1953; 70: 328-331. 